\title{
The role of singular spinor fields in a torsional gravity, Lorentz-violating, framework
}

\author{
A. F. Ferrari, ${ }^{1, *}$ J. A. S. Neto,,${ }^{1,}$ and R. da Rocha ${ }^{1, t+}$ \\ ${ }^{1}$ Universidade Federal do ABC - UFABC, 09210-580, Santo André, SP, Brazil
}

In this work, we consider a generalization of quantum electrodynamics including Lorentz violation and torsional-gravity, in the context of general spinor fields as classified in the Lounesto scheme. Singular spinor fields will be shown to be less sensitive to the Lorentz violation, as far as couplings between the spinor bilinear covariants and torsion are regarded. In addition, we prove that flagpole spinor fields do not admit minimal coupling to the torsion. In general, mass dimension four couplings are deeply affected when singular flagpole - spinors are considered, instead of the usual Dirac spinors. We also construct a mapping between spinors in the covariant framework and spinors in Lorentz symmetry breaking scenarios, showing how one may transliterate spinors of different classes between the two cases. Specific examples concerning the mapping of Dirac spinor fields in Lorentz violating scenarios into flagpole and flag-dipole spinors with full Lorentz invariance (including the cases of Weyl and Majorana spinors) are worked out.

PACS numbers: $04.20 . \mathrm{Gz}, 11.10 .-\mathrm{z}, 03.65 . \mathrm{Pm}$

\footnotetext{
*alysson.ferrari@ufabc.edu.br

† jose.antonio@ufabc.edu.br

¥ roldao.rocha@ufabc.edu.br
} 


\section{INTRODUCTION}

Quantum field theory and particle physics demand that matter is described by fermionic fields. At a first level of classification, previously to second quantization, this means that spinor fields are essential to describe physics. Although Dirac spinors are the most usual objects that carry spinor representations of the Lorentz group, they are actually just the tip of the iceberg that encompasses a comprehensive set of possibilities, described by the Lounesto classification [1, 2], based upon the bilinear covariants. Dirac spinors are included as particular cases of regular spinors, while Majorana and Weyl spinors are well-known examples in the classes of flagpole and dipole spinors, respectively. Besides these, the Lounesto classification also describes a huge class of new possibilities, including mass dimension one spinors [3 6 ], exotic formulations with dynamical mass generation mechanisms [7], and solutions of the Dirac equations in specific circumstances, which are not Dirac spinors. There are still sub-classes in the Lounesto classification which remain unexplored, whose dynamics are still unknown [3, 4, 6]. Reciprocal, equivalent, classifications have further paved recent developments [2, 8, 9]. Many efforts have been devoted to unravel those less known spinors classes. Flagpole spinor include for example Elko and Majorana spinors, and have been used in different contexts, from particle physics and LHC phenomenology to cosmology. Elko spinors have the peculiar feature of being mass dimension one spinor fields (they are not the only ones, as recently pointed out in [6]). As some recent studies regarding flagpole spinors, we can quote those involving: possible signatures via monojets at $14 \mathrm{TeV}$ in $\mathrm{LHC}$ and as a byproduct of a Higgs bubble 10], tunnelling methods and Hawking radiation [11], as well as some cosmological aspects [12, 13, 15 17].

A thorough analysis on the role of flagpoles and flag-dipole spinors and their interpretation in the Penrose formalism was derived in Refs. [1, 3]. New spinors solutions in supergravity have been also recently obtained [18, 19], respectively, in the context of the $\mathrm{AdS}_{4} \times S^{7}$ and $\mathrm{AdS}_{5} \times S^{5}$ compactifications. As a major physical motivation, flagpole spinor fields have been explored as candidates for dark matter $[4,5]$.

Flag-dipoles are spinor fields that were not listed in physics applications until recently, when this class of spinors was shown to provide a solution of the Dirac equation in a $f(R)$, torsional, gravity setup 20]. In fact, in ESK gravity, torsion is coupled to the spin density of the matter field. Therefore, all terms involving the covariant derivatives and the curvature can be split into their torsionless counterparts plus torsional contributions, which can be replaced by the torsion-spin field equations in terms of the spin density of the spinor matter field [12, 13, 15, 20]. ESK theory is 
equivalent to a theory complemented by spin-spin self-interacting potentials, with non-linearities in the matter field equations. The specific gravitational background, for example $f(R)$ or conformal, and the type of spinor (Dirac or other regular spinors; flag-dipoles, flagpoles, or dipole spinors - the singular ones [3]) shall determine the exact structure of these non-linearities in the matter field equations. For example, in the least order derivative ESK gravity with Dirac fermion fields, the non-linearities are realized by axial current squared contact interactions, provided by NambuJona-Lasinio (NJL) potentials. When the spinor field is a flag-dipole fermion field, the interaction is shown to be changed [20].

On the other hand, Lorentz symmetry is the fundamental symmetry underlying the standard model (SM) of particle physics, being experimentally already verified at exquisite level of precision. Nevertheless, the paradigm of Lorentz symmetry might be modified in very high energy regimes. Indeed, whenever quantum gravitational effects can not be dismissed, Lorentz symmetry must be malleable to adequate these extremal regimes into physics. As an example, Lorentz symmetry might be spontaneously broken by some tensorial fields acquiring non-vanishing vacuum expectation values in the low energy effective theory of string theory [21]. A rather general approach in which Lorentz symmetry violation (LV) is incorporated within the Standard Model was developed by Colladay and Kostelecky [22, 23]. This setup has been used as a framework for studying consequences of LV in a plethora of physical phenomena, imposing very stringent bounds on the LV parameters [21, 25 27]. Some LV phenomena that have recently received attention in the literature are effects in acoustic black holes 28], BPS vortices in a background with Lorentz and CPT breaking [29, 31], thick branes in LV [32], among many others.

Our main goal is to study the role of LV in theories containing singular spinor fields in RiemannCartan spacetimes. Since the Palatini and the Einstein-Hilbert Lagrangian densities are physically equivalent, also in the context of the Lounesto classification of spinors, curvature and torsion are equivalent descriptions of the gravitational field. The matter energy-momentum tensor is the source of curvature, in the case of general relativity, and of torsion, in the case of teleparallel gravity. Moreover, torsion-spin coupling can be regarded, besides the curvature-energy coupling. In addition, background torsion violates effective local Lorentz invariance [21]. Riemann-Cartan geometry is the setup for the Einstein-Cartan theory, however, more general gravitation theories in Riemann-Cartan spacetimes may incorporate propagating vierbein and spin-connection fields, describing dynamical torsion and curvature [33].

To consider the torsion coupling to matter fields, usually Dirac fermion fields are employed in the literature. In this setup, torsion effects manifest as self-interactions, capable of providing a 
dynamical explication of the exclusion principle [13]. Gauge couplings on this setup was considered in Ref. [14] and its ramifications. Torsion effects influence the dynamics near or at the Planck scale, wherein breaking of Lorentz invariance could be relevant. Moreover, torsion induces a preferred orientation for a freely falling observer, realized as a manifestation of local Lorentz violation. Hence, constraints on Lorentz symmetry have led to constraints on torsion [21]. We want to analyze the torsion coupling to matter fields beyond Dirac fermion fields, encompassing in the analysis the whole set of singular spinor fields, in particular flagpole and flag-dipole spinor fields. One of our motivations is that recently the Riemann-Cartan geometry shed new light on the prominent roles of singular spinors: it was shown that the Einstein-Sciama-Kibble theory coupled to spinors admits solutions that are not Dirac spinor fields, but flag-dipoles ones [20].

Regardless of its origin in some high energy theory, Lorentz violation is usually accomplished in an effective field theory framework. The so-called Standard Model Extension (SME) [23] contains General Relativity (GR) and the Standard Model (SM). Dominant terms in the SME action include the pure gravity and minimally coupled SM actions, together with all leading-order terms introducing Lorentz symmetry violations that can be constructed from gravitational and SM fields, coupled to constant background tensors. The Riemann-Cartan geometry allows for non-zero vacuum quantities that violate local Lorentz invariance, although preserving general coordinate invariance, encompassing minimal gravitational couplings of spinors. We will show that, in this context, flagpole spinor fields are examples of singular spinor fields that can not couple minimally to torsion.

It is also well known that not all LV parameters in the SME are physically meaningful [22], in the sense that some of them may be absorbed by a field redefinition. Moreover, a properly redefined conserved current satisfies the usual Poincaré algebra, at least as far as these coefficients are concerned. This point was extensively worked out in [34], where a systematic procedure for eliminating spurious LV coefficients and defining conserved currents was developed for the case of QED. The implications of this procedure for theories involving more general types of spinor fields is still lacking, and this is another question we address in this work.

This paper is organized as follows: in Sect.[I] we outline the main setup, briefly introducing the Lounesto classification and essentials on LV and the Riemann-Cartan framework. We shall argue that couplings between the spinor bilinear covariants and torsion are an appropriate signature to probe the sensitiveness of singular spinor fields to LV parameters. We prove that flagpole spinor fields do not admit minimal coupling with torsion, and couplings with mass dimension four coefficients are influenced by the choice of flagpoles spinors, instead of the usual Dirac spinors. In 
Sect.III, the flat spacetime is regarded, and discuss a general mapping between different classes of spinors in LV and Lorentz covariant theories. In particular, we relate regular, Dirac spinors, in a Lorentz violating theory, into singular, flagpole and flag-dipole spinors, in a full Lorentz invariant framework. In Sect.IV the conclusions are presented, further analyzing outlooks and perspectives.

\section{COUPLING SINGULAR SPINORS TO TORSION IN LORENTZ VIOLATING FRAMEWORK}

Let us consider the set of spinor fields in Minkowski spacetime $M \simeq \mathbb{R}^{1,3}$. Given sections of the frame bundle $P_{\mathrm{SO}_{1,3}^{e}}(M)\left\{\mathrm{e}_{\mu}\right\}$, with dual basis $\left\{\mathrm{e}^{\mu}\right\}$, classical spinor fields carrying a $(1 / 2,0) \oplus$ $(0,1 / 2)$ representation of the Lorentz group component connected to the identity $\operatorname{SL}(2, \mathbb{C}) \simeq \operatorname{Spin}_{1,3}^{e}$ are sections of the bundle $P_{\operatorname{Spin}_{1,3}^{e}}(M) \times \mathbb{C}^{4}$. Denoting by $\left\{\gamma^{\mu}\right\}$ the set of gamma matrices, the bilinear covariants are given by

$$
\begin{aligned}
\sigma & =\bar{\psi} \psi \\
\mathbf{J} & =J_{\mu} \mathrm{e}^{\mu}=\bar{\psi} \gamma_{\mu} \psi \mathrm{e}^{\mu} \\
\mathbf{S} & =S_{\mu \nu} \mathrm{e}^{\mu} \wedge \mathrm{e}^{\nu}=\frac{1}{2} \bar{\psi} i \gamma_{\mu \nu} \psi \mathrm{e}^{\mu} \wedge \mathrm{e}^{\nu} \\
\mathbf{K} & =K_{\mu} \mathrm{e}^{\mu}=\bar{\psi} \gamma_{5} \gamma_{\mu} \psi \mathrm{e}^{\mu} \\
\omega & =\bar{\psi} \gamma_{5} \psi
\end{aligned}
$$

where $i \gamma^{5}=\gamma^{0} \gamma^{1} \gamma^{2} \gamma^{3}$. Exclusively in the Dirac theory of the electron, $\mathbf{J}$ is interpreted as being the current density, $\mathbf{K}$ provides the direction of the electron spin, and $\mathbf{S}$ is related to the distribution of intrinsic angular momentum. This physical interpretation is absent in the most general cases. Whenever $\omega=0=\sigma$, the spinor field is said to be singular, otherwise it is a regular spinor. The bilinear covariants for regular spinors satisfy the Fierz identities,

$$
\mathbf{K} \cdot \mathbf{J}=0, \quad \mathbf{S}=\left(\omega+\sigma i \gamma_{5}\right)^{-1} \mathbf{K} \wedge \mathbf{J}, \quad \mathbf{J}^{2}=\omega^{2}+\sigma^{2}=-\mathbf{K}^{2}
$$

Lounesto spinor field classification is based on six disjoint spinor field classes [1],
1) $\sigma \neq 0, \quad \omega \neq 0, \quad \mathbf{J} \neq 0$
4) $\sigma=0=\omega, \quad \mathbf{S} \neq 0, \quad \mathbf{K} \neq 0, \quad \mathbf{J} \neq 0$
2) $\sigma \neq 0, \quad \omega=0, \quad \mathbf{J} \neq 0$
5) $\sigma=0=\omega, \quad \mathbf{S} \neq 0, \quad \mathbf{K}=0, \quad \mathbf{J} \neq 0$
3) $\sigma=0, \quad \omega \neq 0, \quad \mathbf{J} \neq 0$
6) $\sigma=0=\omega, \quad \mathbf{S}=0, \quad \mathbf{K} \neq 0, \quad \mathbf{J} \neq 0$

The first three types of spinor fields, characterized by $\mathbf{J}, \mathbf{K}, \mathbf{S} \neq 0$, are regular. The last three are known, respectively, as flag-dipole, flagpole and dipole spinor fields. It is worth to mention that 
the paradigm of Lounesto classification is concerned with $\mathbf{J} \neq 0$. The mechanism to generate three additional classes has been proposed in [6], including the case $\mathbf{J}=0$, implying that the subsequent spinors have canonical mass dimension one, being conjectured to be ghost fields.

At this point, we review how LV can be introduced in a general way into a theory such as QED, and how some of the LV thus introduced is actually spurious, being eliminated by a proper field redefinition. We shall be interested only in the free fermion piece of the QED Lagrangian, which in the LV case is written as

$$
\mathcal{L}^{\mathrm{LV}-\mathrm{QED}}=\frac{i}{2} \bar{\psi} \Gamma^{\nu} \stackrel{\leftrightarrow}{\nabla_{\nu}} \psi-\bar{\psi} M \psi
$$

where $\nabla_{\mu}=\partial_{\mu}+i q A_{\mu}$ is the covariant derivative, and

$$
\begin{aligned}
\Gamma_{\nu} & :=\gamma_{\nu}+c_{\mu \nu} \gamma^{\mu}+d_{\mu \nu} \gamma_{5} \gamma^{\mu}+\mathfrak{e}_{\nu}+i f_{\nu} \gamma_{5}+\frac{1}{2} g_{\rho \mu \nu} \sigma^{\rho \mu} \\
M & :=m+i m_{5} \gamma_{5}+a_{\mu} \gamma^{\mu}+b_{\mu} \gamma_{5} \gamma^{\mu}+\frac{1}{2} H_{\mu \nu} \sigma^{\mu \nu}
\end{aligned}
$$

$m$ being the electron mass, $\sigma^{\mu \nu}=\frac{i}{2}\left[\gamma^{\mu}, \gamma^{\nu}\right]$, and $a, b, c, d, \mathfrak{e}, f, g, m_{5}, H$ are real constant tensors which parametrize the LV. By assumption, the action (66) is Hermitian, hence constraining the coefficients for Lorentz violation to be real. Some of these parameters have a strong experimental/phenomenological bound, as discussed in [21]. However, some parameters in Eqs. (77) and (8) can be eliminated by a proper field redefinition.

Indeed, a spinor $\chi$ that satisfies a LV extended QED Lagrangian can be obtained from a spinor $\psi$, which is a solution of the standard Lorentz invariant (LI) QED Lagrangian, through the transformation

$$
\psi=\left(\mathbf{1}+f\left(x^{\mu}, \partial_{\nu}\right)\right) \chi=\chi+\left(v \cdot \Gamma+i \mathrm{e}+i \tilde{C}_{\mu} x^{\mu}+C_{\mu \nu} x^{\mu} \partial^{\nu}+B_{\mu} \partial^{\mu}+\gamma_{5} \tilde{B}_{\mu} \partial^{\mu}\right) \chi,
$$

where $f(x, \partial)$ represents a general $4 \times 4$ matrix function of the coordinates and derivatives [34]. Here, $v \cdot \Gamma=v_{I} \Gamma_{I}, \Gamma_{I}$ being a basis for $\mathcal{M}(4, \mathbb{C})$ for a composed index $I \in\{\emptyset, \mu, \mu \nu, \mu \nu \rho, 5\}$, where $\Gamma_{\emptyset}=1$. In addition, $\theta, \tilde{C}_{\mu}, B_{\mu}, \tilde{B}_{\mu}, C_{\mu \nu}$ are scalar coefficients. The parameters $\operatorname{Re}(\theta), B_{\mu}$ and $C_{[\mu \nu]}$ in particular correspond to the $U(1)$ and Poincaré symmetries of the standard Lagrangian. Only lowest order terms in the field redefinition are retained, since the LV parameters are assumed to be small. These redefinitions can be regarded as position-dependent mixings of components in spinor space.

For simplicity, we quote the result of the redefinition parametrized by the parameter $v$, which have been first described in [22]. We start with an explicitly LI Lagrangian

$$
\mathcal{L}=\frac{i}{2} \bar{\psi} \gamma^{\mu} \stackrel{\leftrightarrow}{\partial_{\mu}} \psi-m \bar{\psi} \psi
$$


which is rewritten via the redefinition (9) as

$$
\mathcal{L}=\frac{i}{2} \bar{\chi} \gamma^{\mu} \stackrel{\leftrightarrow}{\partial_{\mu}} \chi-m \bar{\chi} \chi+\frac{i}{2} \bar{\chi}\left[\left\{\gamma^{\mu}, \Gamma \cdot \operatorname{Re} v\right\}+i\left[\gamma^{\mu}, \Gamma \cdot \operatorname{Im} v\right]\right] \overleftrightarrow{\partial_{\mu}} \chi-2 m \operatorname{Re} v \cdot \bar{\chi} \Gamma \chi
$$

The essential point is that both Lagrangians describe the same physics, so even if Eq. (11) includes terms that might seem to violate Lorentz invariance, this theory is actually Lorentz invariant. Indeed, one can define properly modified Poincaré generators in terms of $\chi$ that satisfy the usual Poincaré algebra. This example shows that the general field redefinition described by Eq. (9) can eliminate some of the coefficients in a general LV effective theory as the SME, since these coefficients are actually unobservable at leading order in SME-related phenomenology.

It is worth to emphasize that Lagrangians having derivative terms for spinors are here adopted. Hence, the field equations have terms given by the Dirac operator and terms that have a similar differential order that influence the dynamics and the light-cone structure as well [35, 36]. Interactions that have the same derivative order of the leading kinetic term can yield pathologies like the mismatch between degrees of freedom and field equations and superluminal propagation, among others. In general, matter fields are classified according to their spin [37]. In fact, matter fields have degrees of freedom, corresponding to solutions of systems of differential equations, yielding the highest-order time derivative for the field. Constraints that obstruct the appearance of such highest-order time derivatives should, thus, be imposed [37]. For higher-spin matter fields, inconsistencies can be circumvented by the Velo-Zwanziger procedure [35, 36]. In Ref. [36] a similar prescription is employed to introduce an appropriate Dirac operator. The Velo-Zwanziger problem has been generalized to encompass torsion [39 41] and the torsion-spin coupling [42]. The constraints imposed by the Velo-Zwanziger analysis are shown to be strengthened by the backreaction due to the torsion, having also further constraints coming from the spin-torsion coupling. These constraints can avoid the existence of intricate matter fields, restricting the amount of fields available to be used in this kind of theory.

To simplify further, we assume $v \cdot \Gamma=v_{\mu} \gamma^{\mu}$. Denoting the conventional free field Lagrangian for $\chi$ by

$$
\mathcal{L}_{0}=\frac{i}{2} \bar{\chi} \Gamma^{\nu} \stackrel{\leftrightarrow}{\partial_{\nu}} \chi-m \bar{\chi} \chi
$$

equation (마) can be written as,

$$
\mathcal{L}=\mathcal{L}_{0}+\operatorname{Re} v_{\mu}\left[i \bar{\chi} \overleftrightarrow{\partial^{\mu}} \chi-2 m \bar{\chi} \gamma^{\mu} \chi\right]-i \operatorname{Im} v_{\mu}\left[\bar{\chi} \sigma^{\mu \nu} \stackrel{\leftrightarrow}{\partial_{\nu}} \chi\right]
$$

Comparing with (7), the simultaneous choice of LV parameters $\mathfrak{e}^{\mu}=2 \operatorname{Re} v^{\mu}$ and $a^{\mu}=2 m \operatorname{Re} v^{\mu}$ may be entirely attributed to the field redefinition, and therefore does not introduce a real LV. 
In a different perspective, one might say that in the LV theory defined by (6), either $\mathfrak{e}^{\mu}$ or $a^{\mu}$ can be eliminated via a field redefinition. The term $\operatorname{Im} v_{\mu}$ indicates that the choice $2 \operatorname{Im} v_{[\mu} g_{\rho] \nu}=g_{\rho \mu \nu}$ eliminates the terms proportional to $g_{\rho \mu \nu}$ in (6). The parameters $m_{5}, a_{\mu}, \mathfrak{e}_{\mu}, f_{\mu}$, and $c_{[\mu \nu]}$ in (6) can also be removed [34].

Now we review how this discussion can be extended to the Riemann-Cartan torsional context. By using latin indexes to label local Lorentz coordinates and greek indexes for spacetime ones, the Minkowski metric is related to the curved-spacetime metric $g_{\mu \nu}$ via the vierbein $e_{\mu}{ }^{a}$, by the relation $g_{\mu \nu}=e_{\mu}{ }^{a} e_{\nu}{ }^{b} \eta_{a b}$. The determinant of the vierbein is denoted by $e$ and the charge of the electron is denoted by $-q$. For the spacetime covariant derivative, the connection is assumed to be metric compatible. In addition, curved-spacetime indexes are corrected with the Cartan connection $\Gamma_{\mu \nu}^{\lambda}$, namely,

$$
\nabla_{\mu} e_{\nu}^{a}=\partial_{\mu} e_{\nu}^{a}+\omega_{\mu b}^{a} e_{\nu}^{b}-\Gamma_{\mu \nu}^{\alpha} e_{\alpha}^{a}
$$

The contortion tensor is defined as

$$
K_{\mu \nu}^{\lambda}=\frac{1}{2}\left(T_{\mu \nu}^{\lambda}-T_{\nu \mu}^{\lambda}-T_{\mu \nu}^{\lambda}\right)
$$

and the curvature as $R_{\lambda \mu \nu}^{\kappa}=\stackrel{\circ}{R}_{\lambda \mu \nu}^{\kappa}+\nabla_{[\mu} K_{\nu] \lambda}^{\kappa}+K_{[\mu \nu]}^{\alpha} K_{\alpha \lambda}^{\kappa}+K_{[\mu \lambda}^{\alpha} K_{\nu] \alpha}^{\kappa}$, where $\stackrel{\circ}{R}_{\lambda \mu \nu}^{\kappa}$ denotes the usual Riemann curvature tensor in the absence of torsion. The source of contortion may be considered as a Kalb-Ramond field $B_{\alpha \beta}$, through $K_{\alpha \beta}^{\rho}=-\frac{1}{\kappa^{3 / 2}} H_{\alpha \beta}^{\rho}$, where $H_{\rho \alpha \beta}=\partial_{[\rho} B_{\alpha \beta]}$ and $\kappa$ denotes the coupling constant. However, our discussion will not depend on this identification, being a generic geometric contortion considered hereon.

Signatures of torsion, in the context of both minimal and non-minimal couplings to fermions, are phenomenologically and experimentally abundant [21]. The minimal coupling between torsion and SM fields is realized through covariant derivatives. Nevertheless, non-minimal couplings are also an option. In fact, we shall prove that when flagpoles spinors are regarded, non-minimal couplings are the only possibility.

The essential variables here are the vierbein and the spin connection, since other variables such as curvature and torsion can be expressed in terms of these. For example, the Cartan connection reads $\Gamma_{\mu \nu}^{\lambda}=e^{\lambda a}\left(\partial_{\mu} e_{\nu a}-\omega_{\mu a}^{b} e_{\nu b}\right)$, whereas the torsion is given by $T_{\lambda \mu \nu}=e_{\lambda}^{a}\left(\partial_{[\mu} e_{\nu] a}+\omega_{[\mu|a b|} e_{\nu]}^{b}\right)$. Moreover, the spin connection is related to the vierbein by

$$
\omega_{\mu}^{a b}=\frac{1}{2} e^{\nu[a} \partial_{[\mu} e_{\nu]}^{b]}-\frac{1}{2} e^{\alpha a} e^{\beta b} e_{\mu}^{c} \partial_{[\alpha} e_{\beta] c}+K_{\nu \mu \lambda} e^{\nu a} e^{\lambda b}
$$

Hereon weak gravitational fields, $g_{\mu \nu}=\eta_{\mu \nu}+h_{\mu \nu}$, are regarded, where $h_{\mu \nu}$ is a fluctuation. At 
leading order, the vierbein and spin connection can be expressed in terms of small quantities,

$$
\begin{aligned}
e_{\mu a} & =\eta_{\mu a}+\epsilon_{\mu a} \approx \eta_{\mu a}+\frac{1}{2} h_{\mu a}+\chi_{\mu a}, \quad e \approx 1+\frac{1}{2} h, \\
\omega_{\mu a b} & \approx-\frac{1}{2} \partial_{a} h_{\mu b}+\frac{1}{2} \partial_{b} h_{\mu a}+\partial_{\mu} \chi_{a b}+K_{a \mu b} .
\end{aligned}
$$

The basic non-gravitational fields for the Lorentz- and CPT-violating QED extension in Riemann-Cartan spacetime are a fermion field $\psi$ and the photon field $A_{\mu}$. The action for the theory can be expressed as a sum of partial actions for the fermion, for the photon and for gravity. The fermion part of the action contains terms that are dominant at low energies, involving fermions and their minimal couplings to photons and gravity. In general, we also have to consider higher order terms involving fermions and photons that are non-renormalizable, non-minimal and higher order in the gravitational couplings, as well as field operators of dimension greater than four that couple curvature and torsion to the matter and photon fields.

The fermion part of the action (6) for the QED extension can be written as

$$
S=\int d^{4} x e\left(\frac{1}{2} i e_{a}^{\mu} \bar{\psi} \Gamma^{a} \stackrel{\leftrightarrow}{D_{\mu}} \psi-\bar{\psi} M \psi\right)
$$

where the usual $U(1)$ covariant derivative is given by $\nabla_{\mu} \psi \equiv \partial_{\mu} \psi+\frac{1}{4} i \omega_{\mu}^{a b} \sigma_{a b} \psi-i q A_{\mu} \psi[15]$. In addition, Eqs. (7) and (86) read, respectively, in terms of multivector structure of the vierbein,

$$
\begin{aligned}
& \Gamma^{a}=\gamma^{a}-c_{\mu \nu} e^{\nu a} e^{\mu}{ }_{b} \gamma^{b}+d_{\mu \nu} e^{\mu}{ }_{b} e^{\nu a} \gamma^{b} \gamma_{5}-\mathfrak{e}_{\rho} e^{\rho a}-i f_{\mu} \gamma_{5} e^{\mu a}-\frac{1}{2} g_{\lambda \mu \nu} e_{b}^{\lambda} e^{\nu a} \mathfrak{e}^{\mu} c \sigma^{b c}, \\
& M=m+a_{\mu} e^{\mu}{ }_{a} \gamma^{a}+\frac{1}{2} H_{\mu \nu} e_{a}^{\mu} e^{\nu}{ }_{b} \sigma^{a b}+b_{\mu} e^{\mu}{ }_{a} \gamma_{5} \gamma^{a}+i m_{5} \gamma_{5} .
\end{aligned}
$$

Hence the Dirac equation in Riemann-Cartan spacetimes reads [34]

$$
i e^{\mu}{ }_{b} \Gamma^{b} \nabla_{\mu} \psi+\frac{1}{2} e^{\mu}{ }_{a} \omega_{\mu}^{b c}\left(i \eta_{b}^{a} \Gamma_{c}-\frac{1}{4}\left[\sigma_{b c}, \Gamma^{a}\right]\right) \psi-\frac{1}{2} i T_{\rho \mu}^{\rho} e^{\mu}{ }_{a} \Gamma^{a} \psi-M \psi=0 .
$$

The LV terms involving $M$ contribute to the Dirac equation in a minimal way, as assumed in nonderivative couplings. Nevertheless, those terms involving $\Gamma^{a}$ emerge both minimally and through commutation with the Lorentz generators in the covariant derivative. In particular, the Lorentzinvariant parts of the terms in Eq. (22) cancel out.

Lorentz violating corrections can be then explored in the context of singular spinor fields. In fact, the QED extension in Minkowski and Riemann-Cartan spacetimes differ by weak gravitational couplings. In this regime, given by Eq. (18), the Lagrangian terms that are linear read

$$
\mathcal{L}_{\psi} \sim-i\left(c_{\text {effective }}\right)_{\mu \nu} \bar{\psi} \gamma^{\mu} \partial^{\nu} \psi-\left(b_{\text {effective }}\right)_{\mu} \overbrace{\bar{\psi} \gamma_{5} \gamma^{\mu} \psi}^{=K^{\mu}},
$$


where

$$
\left(c_{\text {effective }}\right)_{\mu \nu} \equiv c_{\mu \nu}+\chi_{\mu \nu}-\frac{1}{2} h_{\mu \nu}, \quad\left(b_{\text {effective }}\right)_{\mu} \equiv b_{\mu}+\frac{1}{8} \epsilon_{\mu \nu \rho \sigma} T^{\nu \rho \sigma}-\frac{1}{4} \partial^{\nu} \chi^{\rho \sigma} \epsilon_{\mu \nu \rho \sigma} .
$$

In this expression, leading-order terms arising from the scaling of the vierbein determinant $e$ are neglected, for being LI. Equations (24) show that, at leading order, a weak background metric is governed by $c_{\mu \nu}$, whereas the torsion is effectively ruled by a $b_{\mu}$ term [43]. The latter is a CPT-violating term, so the presence of background torsion can mimic CPT violation. If the Lagrangian (24) models a flagpole spinor field, that satisfies $K^{\mu}=\bar{\psi} \gamma_{5} \gamma^{\mu} \psi=0$, the $b_{\text {effective term }}$ is irrelevant in such a model. Hence, flagpole fermions are not sensitive to this type of LV.

Considering the mapping between LI and LV spinor fields given by Eq. (91), they can be used to show that, at leading order in coefficients for Lorentz violation, there are no physical effects from the coefficients $\mathfrak{e}_{\mu}, f_{\mu}$, or from the antisymmetric parts of $c_{\mu \nu}$.

When non-minimal gravitational couplings are taken into account, operators of mass dimension four or less can be analyzed. In the QED extension, such non-minimal operators are not leading couplings, and the only gauge-invariant ones are products of the torsion with fermion bilinear covariants. The Lorentz invariant possibilities are

$$
\mathcal{L}_{\mathrm{LI}}=\left(a T_{\lambda \mu}^{\lambda}+a_{5} \epsilon_{\mu \nu \rho} T^{\mu \nu \rho}\right) \overbrace{\bar{\psi} \gamma^{\mu} \psi}^{=J^{\mu}}+\left(b T_{\lambda \sigma}^{\lambda}+b_{5} T^{\mu \nu \rho} \epsilon_{\mu \nu \rho \sigma}\right) \overbrace{\bar{\psi} \gamma_{5} \gamma^{\mu} \psi}^{=K^{\sigma}} .
$$

The $b_{5}$ coupling is minimal, whereas the other ones are non minimal. The LV possibilities are

$$
\begin{aligned}
\mathcal{L}_{\mathrm{LV}}=k_{\mu \nu \rho} T^{\mu \nu \rho} & \overbrace{\bar{\psi} \psi}^{=\sigma}+k_{\mu \nu \rho \sigma} T^{\mu \nu \rho} \overbrace{\bar{\psi} \gamma^{\sigma} \psi}^{=J^{\sigma}}+k_{\mu \nu \rho \sigma \tau} T^{\mu \nu \rho} \overbrace{\bar{\psi} \sigma^{\sigma \tau} \psi}^{=-2 i S^{\sigma \tau}}+k_{5 \mu \nu \rho \sigma} T^{\mu \nu \rho} \overbrace{\bar{\psi} \gamma_{5} \gamma^{\sigma} \psi}^{K^{\sigma}} \\
& +k_{5 \mu \nu \rho} T^{\mu \nu \rho} \overbrace{\bar{\psi} \gamma_{5} \psi}^{\omega} .
\end{aligned}
$$

The $k_{\mu \gamma \alpha \beta}$ must have the symmetries of the Riemann tensor [23]. In our case, we use the equation (33) of [24], with the following form $k_{\mu \gamma \alpha \beta}=\frac{1}{2}\left(\eta_{\mu \alpha} h_{\gamma \beta}-\eta_{\gamma \alpha} h_{\mu \beta}+\eta_{\gamma \beta} h_{\mu \alpha}-\eta_{\mu \beta} h_{\gamma \alpha}\right)$, where $h_{\mu \nu}$ is the weak-field background metric. If Lorentz violation is suppressed, and the torsion is also small, then all terms in Eq. (26) are subdominant. Nonetheless, all the above operators may be of interest in more exotic scenarios. For example, the presence of a Higgs doublet in the SME allows for other types of non-minimal gravitational couplings of dimension four or more, including ones involving both curvature and torsion. Operators of dimension greater than four generically come with Planck-scale suppression [21]. Therefore, effects of dimension five Lorentz invariant operators suppressed by the inverse of the Planck mass $m_{P}$ are comparable, in magnitude, to those of a dimension four operator involving a coefficient for LV suppressed by $m_{P}$, for example. 
It is worth to emphasize that, when flagpole spinor fields are taken into account, the second and fourth terms on the right-hand side of Eq. (26) identically vanish, as well as the second and fourth terms on the right-hand side of Eq. (25) . Hence, flagpole fermions have a restricted range of couplings, when compared to Dirac fermions.

By taking into account the Lounesto classification, we already know that in Riemann-Cartan geometry in a $f(R)$ conformal gravity setup, flag-dipole type-(4) spinor fields are solutions of the Dirac equation [20]. In this context, flagpole also play a prominent role when torsion is taken into account, as the last term on the right hand side in Eq. (25) is zero, as well as the first, the second, and the last term on the right hand side in Eq. (26). Hence, flagpole spinor fields are examples of singular spinor fields which we have proved to be less sensitive to Lorentz violation.

Fermionic matter fields in Riemann-Cartan spacetimes can be governed by a Lagrangian with arbitrary torsion couplings. In a constant torsion approximation setup, the torsion couplings can be replaced by background solutions to the torsion field equations. The corresponding effective Lagrange density reads [21]

$$
\mathcal{L} \sim \frac{1}{2} i \bar{\psi} \gamma^{\mu} \stackrel{\leftrightarrow}{\partial_{\mu}} \psi-m \bar{\psi} \psi+\mathcal{L}_{\mathrm{LI}(4)}+\mathcal{L}_{\mathrm{LI}(5)}
$$

where all possible independent constant-torsion couplings of mass dimensions four and five are respectively given by

$$
\begin{aligned}
\mathcal{L}_{\mathrm{LI}(4)} & =\left(a_{1} T_{\rho \mu}^{\rho}+a_{3} \mathfrak{A}_{\mu}\right) \overbrace{\bar{\psi} \gamma^{\mu} \psi}^{=J^{\mu}}+\left(a_{2} T_{\rho \mu}^{\rho}+a_{4} \mathfrak{A}_{\mu}\right) \overbrace{\bar{\psi} \gamma_{5} \gamma^{\mu} \psi}^{=K^{\mu}}+\frac{1}{2} i \stackrel{\circ}{a}_{1} T^{\mu} \bar{\psi} \stackrel{\leftrightarrow}{\partial}_{\mu} \psi \\
\mathcal{L}_{\mathrm{LI}(5)} & =\frac{1}{2}\left(\stackrel{\circ}{a}_{2} T^{\mu}+\stackrel{\circ}{a}_{4} \mathfrak{A}^{\mu}\right) \bar{\psi} \gamma_{5}{\stackrel{\leftrightarrow}{\partial_{\mu}}}_{\mu} \psi+\frac{1}{2} i \stackrel{\circ}{a}_{3} \mathfrak{A}^{\mu} \bar{\psi} \stackrel{\leftrightarrow}{\partial_{\mu}} \psi+\frac{1}{2} i\left(\stackrel{\circ}{a}_{5} M_{\mu \lambda}^{\nu}+\stackrel{\circ}{a}_{6} T_{\rho \mu}^{\rho}+\stackrel{\circ}{a}_{7} \mathfrak{A}_{\mu}\right) \bar{\psi}{\stackrel{\leftrightarrow}{\partial_{\nu}}}_{\nu}^{\mu \nu} \psi \\
& +\frac{1}{2} i\left(\stackrel{\circ}{a}_{8} \epsilon^{\lambda \kappa \mu \nu} T_{\rho \lambda}^{\rho}+\stackrel{\circ}{a}_{9} \epsilon^{\lambda \kappa \mu \nu} \mathfrak{A}_{\lambda}\right) \bar{\psi}{\stackrel{\leftrightarrow}{\partial_{\kappa}}}_{\sigma_{\mu \nu}} \psi
\end{aligned}
$$

where the $a^{A}\left[\stackrel{\circ}{a}^{A}\right]$ denote mass dimension four [five] coupling constants,

$$
M_{\alpha \mu \nu}=\frac{1}{3}\left(T_{\alpha \mu \nu}+T_{\mu \alpha \nu}+T_{\mu} g_{\alpha \mu}\right)-\frac{1}{3}(\mu \leftrightarrow \nu),
$$

and $\mathfrak{A}^{\mu}=\frac{1}{6} \epsilon^{\alpha \beta \gamma \mu} T_{\alpha \beta \gamma}[21$, 24].

It is worth to emphasize that the Lagrangian in Eq. (28) is gauge invariant, whereas Eq. (31) is not gauge invariant. The minimal coupling is obtained in the particular case where $a_{4}=3 / 4$ and all other couplings vanish. By considering Eqs. (3) to (5), we can see that type-(5), flagpole spinor fields, are described by

$$
\mathcal{L}_{(4)}^{\text {type-(5) }} \sim\left(a_{1} T_{\rho \mu}^{\rho}+a_{3} \mathfrak{A}_{\mu}\right) \overbrace{\bar{\psi} \gamma^{\mu} \psi}^{=J^{\mu}},
$$


and at least half of the couplings between flagpole fermions, associated with dimension four coupling constants, and torsion, are vanishing. We have shown that, in Riemann-Cartan spacetimes, flagpoles spinors are less sensitive to Lorentz violation. Recent experimental searches for Lorentz violation are exploited to extract new constraints involving independent torsion components down to levels of order $10^{-31} \mathrm{GeV}$ [21]. Although exceptional sensitivity to spacetime torsion can be achieved by searching for its couplings to Dirac fermions, flagpole spinors are shown to be less sensitive to torsion.

\section{A BRIDGE BETWEEN LORENTZ SYMMETRY AND LORENTZ SYMMETRY VIOLATION}

Once we proved that singular spinors are less sensitive to torsion couplings in LV scenarios, we now discuss the relation between bilinear covariant in the apparently LV framework and the standard covariant bilinears. Since bilinear covariants realize the observables in theories involving fermionic fields, this is a question of upmost physical relevance. We will discuss how a general LV spinor field can be transliterated into the Lorentz-invariant ones, and show that this relation mixes the spinor classes as defined in the Lounesto classification.

One formal concern when considering LV is what part of the Lorentz symmetry is broken and what part does remain. Hence, if LV is obtained by allowing a larger class of transformations that are, in general, non-linear and dependent on coordinates and derivatives, this formalism should embed the Lorentz group into a larger group. This might make it necessary to describe how the Lorentz group places inside this larger symmetry group. However, by focusing on the study of spinors via the bilinear invariants, we do not need to address these questions. In fact, bilinear covariants of any spinor does concern neither to the representation with respect to the residual symmetry nor to the group associated with the residual symmetry. This is due to the fact that any symmetry group can be embedded in some Spin group that, up to dimension five, can be defined by the invertible elements $R$ of the twisted Clifford-Lipschitz group that satisfy $R^{\dagger} R=I$ [1, 2]. Hence, any residual symmetry shall not be apparent when bilinear covariants are taken into account. When spinors are taken into account, then obviously the content of the residual symmetry is important. Nevertheless, our aim to take into account the observables, namely the bilinear covariants, make the group content to be shortcut. It is worth to mention that by taking a classical spinor $\xi$ which satisfies $\xi^{\dagger} \gamma_{0} \psi \neq 0$, the original spinor $\psi$ can be recovered from its aggregate $\mathbf{Z}$, which is given by

$$
\mathbf{Z}=\sigma+\mathbf{J}+i \mathbf{S}+i \mathbf{K} \gamma_{0123}+\omega \gamma_{0123}
$$


using the Takahashi algorithm [2].

Besides removing spurious Lorentz violation terms from the Lagrangian, we want to investigate the effect of these field redefinitions in the Lounesto classification for the transformed spinors. We accomplish this by relating the observables in the Lorentz violating framework with the bilinear covariants in the standard LI theory. Hence the regular (in particular Dirac) spinors and the singular spinors (encompassing Weyl, Majorana and Elko spinors, among others) can have a dual description in terms of a Lorentz violating structure.

We start by listing the covariant bilinears in the Lorentz violating framework,

$$
\begin{aligned}
\sigma_{\chi} & =\bar{\chi} \chi \\
\mathbf{J}_{\chi} & =\bar{\chi} \gamma_{\mu} \chi \mathrm{e}^{\mu}, \\
\mathbf{S}_{\chi} & =\frac{1}{2} \bar{\chi} i \gamma_{\mu \nu} \chi \mathrm{e}^{\mu} \wedge \mathrm{e}^{\nu}, \\
\mathbf{K}_{\chi} & =\bar{\chi} \gamma_{5} \gamma_{\mu} \chi \mathrm{e}^{\mu}, \\
\omega_{\chi} & =\bar{\chi} \gamma_{5} \chi .
\end{aligned}
$$

Next, we relate these invariants with the corresponding ones for the transformed (LI) spinors. After some calculation, we find

$$
\sigma_{\psi}=\Delta \sigma_{\chi}+\Omega_{\sigma}
$$

where $\Delta=1+2 \operatorname{Im} \theta+|\theta|^{2}+2 \tilde{C}_{\mu} x^{\mu} \operatorname{Re} \theta+\tilde{C}_{\mu} \tilde{C}_{\nu} x^{\mu} x^{\nu}$, and the explicit form of $\Omega_{\sigma}$ is given in the Appendix. The first terms of this expression read

$$
\Omega_{\sigma}=\chi^{\dagger} \gamma_{0} v \cdot \Gamma \chi+B_{\mu} \chi^{\dagger} \gamma_{0} \partial^{\mu} \chi+\tilde{B}_{\mu} \chi^{\dagger} \gamma_{0} \gamma_{5} \partial^{\mu} \chi+\cdots
$$

For the remaining invariants we obtain similar expressions,

$$
\begin{aligned}
J_{\alpha}^{\psi} & =\Delta J_{\alpha}^{\chi}+\Omega_{J_{\alpha}},\left(\gamma_{0} \mapsto \gamma_{0} \gamma_{\alpha}\right) \\
S_{\alpha \beta}^{\psi} & =\Delta S_{\alpha \beta}^{\chi}+\Omega_{S_{\alpha \beta}},\left(\gamma_{0} \mapsto \gamma_{0} \gamma_{\alpha \beta}\right) \\
K_{\alpha}^{\psi} & =\Delta K_{\alpha}^{\chi}+\Omega_{K_{\alpha}},\left(\gamma_{0} \mapsto \gamma_{0} \gamma_{5} \gamma_{\alpha}\right) \\
\omega^{\psi} & =\Delta \omega^{\chi}+\Omega_{\omega},\left(\gamma_{0} \mapsto \gamma_{0} \gamma_{5}\right)
\end{aligned}
$$

where the identifications in the parentheses mean that, for example, $\Omega_{J_{\alpha}}$ is obtained from $\Omega_{\sigma}$ by the substitution $\gamma_{0} \mapsto \gamma_{0} \gamma_{\alpha}$, and similarly for the remaining invariants.

Using these expressions, we can apply the Lounesto classification for the transformed (LI) spinors. Consider for example the case $\sigma_{\psi} \neq 0, \omega_{\psi} \neq 0$, corresponding to a type- 1 spinor in the LI 
theory. Since $\sigma_{\psi}=\Delta \sigma_{\chi}+\Omega_{\sigma}$ and $\omega_{\psi}=\Delta \omega_{\chi}+\Omega_{\omega}$, depending on the values of $\Omega_{\sigma}$ and $\Omega_{\omega}$, one can have either $\sigma_{\chi}$ and $\sigma_{\omega}$ equal to zero or not, and therefore the field redefinition may relate a Lorentz invariant type-1 spinor to several types of Lorentz violating spinors, such as $\sigma_{\chi}=0=\omega_{\chi}$ (types 4, 5, and 6), and others. A comprehensive list of possibilities is given below, where we shall assume that the functions $\Delta$ and $\Omega$ are non-vanishing:

$\left.1_{\psi}\right) \sigma_{\psi} \neq 0, \quad \omega_{\psi} \neq 0$

Since $\sigma_{\psi} \neq 0$ and $\sigma_{\psi}=\Delta \sigma_{\chi}+\Omega_{\sigma}$, we list below all possibilities, depending upon whether $\sigma_{\chi}$ either does or does not equal zero, as well as $\omega_{\chi}$ :

i) $\sigma_{\chi}=0=\omega_{\chi}$. These conditions are correspondent to the type-(4), type-(5), and type-(6) spinor fields - respectively flag-dipoles, flagpoles, and dipoles.

ii) $\sigma_{\chi}=0$ and $\omega_{\chi} \neq 0$, being compatible to type-(3) regular spinor fields. The condition $\sigma_{\chi}=0$ is consistent with $\sigma_{\psi} \neq 0$.

iii) $\sigma_{\chi} \neq 0$ and $\omega_{\chi}=0$. This case regards type-(2) regular spinor fields. The condition $\omega_{\chi}=0$. is consistent with $\omega_{\sigma} \neq 0$

iv) $\sigma_{\chi} \neq 0$ and $\omega_{\chi} \neq 0$, corresponding to type-(1) Dirac spinor fields.

$\left.2_{\psi}\right) \sigma_{\psi} \neq 0, \quad \omega_{\psi}=0$.

Although the condition $\sigma_{\psi} \neq 0$ is consistent with both the possibilities $\sigma_{\chi}=0$ and $\sigma_{\chi} \neq 0$ (clearly the condition $\sigma_{\chi} \neq 0$ is consistent with $\sigma_{\psi} \neq 0$ if $\Delta \sigma_{\chi} \neq-\Omega_{\sigma}$ ), the condition $\omega_{\psi}=0$ yields $\Delta \omega_{\chi}=-\Omega_{\sigma}$, which does not vanish.

i) $\sigma_{\chi}=0$ and $\omega_{\chi} \neq 0$. This case corresponds to the type-(3) regular spinor fields. The condition $\sigma_{\chi}=0$ is consistent with $\sigma_{\psi} \neq 0$, however since $\omega_{\chi} \neq 0$, the additional condition $\Delta \omega_{\chi}=-\Omega_{\sigma}$ must be imposed.

ii) $\sigma_{\chi} \neq 0$ and $\omega_{\chi} \neq 0$. This case regards type-(1) Dirac spinor fields.

$\left.3_{\psi}\right) \sigma_{\psi}=0, \quad \omega_{\psi} \neq 0$. The condition $\omega_{\psi} \neq 0$ is consistent with both complementary $\omega_{\chi}=0$ and $\omega_{\chi} \neq 0$

To summarize:

i) $\omega_{\chi}=0$ and $\sigma_{\chi} \neq 0$, corresponding to type-(2) regular spinor fields. The condition $\omega_{\chi}=0$ is consistent with $\omega_{\psi} \neq 0$, however since $\sigma_{\chi} \neq 0$, the additional condition $\sigma_{\psi}=\Delta \sigma_{\chi}+\Omega_{\sigma} \neq 0$ must be imposed. 


\begin{tabular}{||c|r||c|c||}
\hline & LV Spinor Fields & Covariant Spinor Fields & \\
\hline \hline class $\left(1_{\Psi}\right)$ & $\Psi$-regular & Regular & class (1) \\
& & Regular & class (2) \\
& & Regular & class (3) \\
& & Flag-dipoles & class (4) \\
& & Flagpoles & class (5) \\
& & Dipoles & class (6) \\
& & Regular & class (3) \\
& & Regular & class (1) \\
\hline class $\left(2_{\Psi}\right)$ & $\Psi$-regular & Regular & class (2) \\
& & Regular & class (1) \\
\hline class $\left(3_{\Psi}\right)$ & $\Psi$-regular & Regular & class (1) \\
& & Regular & class (1) \\
\hline class $\left(4_{\Psi}\right)$ & $\Psi$-flag-dipole & Regular & class (1) \\
\hline class $\left(5_{\Psi}\right)$ & $\Psi$-flagpole & $\Psi$-dipole &
\end{tabular}

TABLE I. Correspondence among LV spinor fields $(\chi)$ and the LI $\Psi$-spinor fields under Lounesto spinor field classification.

ii) $\sigma_{\chi} \neq 0$ and $\omega_{\chi} \neq 0$. This case regards type-(1) Dirac spinor fields.

$$
\begin{aligned}
& \left.4_{\psi}\right) \sigma_{\psi}=0=\omega_{\psi}, \quad \mathbf{K}_{\psi} \neq 0, \quad \mathbf{S}_{\psi} \neq 0 . \\
& \left.5_{\psi}\right) \sigma_{\psi}=0=\omega_{\psi}, \quad \mathbf{K}_{\psi}=0, \quad \mathbf{S}_{\psi} \neq 0 . \\
& \left.6_{\psi}\right) \quad \sigma_{\psi}=0=\omega_{\psi}, \quad \mathbf{K}_{\psi} \neq 0, \quad \mathbf{S}_{\psi}=0 .
\end{aligned}
$$

All singular spinor fields $\left.4_{\psi}\right), 5_{\psi}$ ), and $6_{\psi}$ ) are defined by the condition $\sigma_{\psi}=0=\omega_{\psi}$, which implies that $\Delta \sigma_{\chi}=-\Omega_{\sigma}(\neq 0)$ and that $\Delta \omega_{\chi}=-\Omega_{\omega}(\neq 0)$. Therefore, singular spinors in the Lorentz violating theory are always related to a regular spinor in the corresponding Lorentz invariant model. This discussion can be summarized in Table【 that describes the possibility of mapping observables in the Lorentz violating framework into observables in the Lorentz covariant models.

The possibility of mapping between different spinor classes has already been pointed out in the literature. In fact, it is known that a regular spinor field can be mapped into any covariant spinor field, including exotic mappings [7]. Such mappings are formally consistent and were accomplished both in the kinematical and the dynamical contexts. Nevertheless, all these studies were developed under a Lorentz invariant framework, that is generalized in this present work to include the possibility of LV, thus enlarging considerably the class of models that can be related by these transformations. 
As an example of the kind of relation discussed here, let us construct a transformation that maps a Dirac spinor $\chi$ in the LV framework to a singular spinor $\psi$ (Majorana, flagpole, type) in the LI one. Without loss of generality, in the Weyl representation these spinors are eigenspinors of the charge conjugation operator and can be parametrized as follows,

$$
\chi=\left(\begin{array}{l}
a_{0} \\
a_{1} \\
a_{2} \\
a_{3}
\end{array}\right)=\left(\begin{array}{l}
\chi_{1} \\
\chi_{2}
\end{array}\right) \in \mathbb{C}^{4}, \quad \psi=\left(\begin{array}{c}
-i \beta^{*} \\
i \alpha^{*} \\
\alpha \\
\beta
\end{array}\right)=\left(\begin{array}{c}
\psi_{1} \\
\psi_{2}
\end{array}\right) \in \mathbb{C}^{4},
$$

and we can further choose $a_{0}=a_{2}=0$ [8]. The necessary transformation is of the form $\psi=$ $\left(1+v_{\mu} \gamma_{5} \gamma^{\mu}\right) \chi$, which is equivalent to $\psi-\chi=v_{\mu} \gamma_{5} \gamma^{\mu} \chi$. Defining

$$
T \equiv v_{\mu} \gamma_{5} \gamma^{\mu}=\left(\begin{array}{cc}
0 & -v_{\mu} \sigma^{\mu} \\
v_{0} I_{2}-v_{k} \sigma^{k} & 0
\end{array}\right)
$$

where $\sigma^{0}=I_{2}$, and $\sigma^{k}$ are the Pauli matrices, namely, $T \chi=\left(\begin{array}{c}-v_{\mu} \sigma^{\mu}\left(\chi_{2}\right) \\ v_{0} I-v_{k} \sigma^{k}\left(\chi_{1}\right)\end{array}\right)=\left(\begin{array}{c}\psi_{1}-\chi_{1} \\ \psi_{2}-\chi_{2}\end{array}\right)$. Hence we can obtain consistency conditions between the components of the spinors, namely, $i \beta^{*} / a_{3}=$ $-\alpha / a_{1} \equiv \delta \in \mathbb{C}$, together with $v_{1}-i v_{2}=\delta$, which characterizes the desired transformations. The end result is that, in the classical level, the LV model of a Dirac spinor $\chi$ governed by the Lagrangian

$$
\mathcal{L}_{0}=\frac{i}{2} \bar{\chi} \gamma^{\nu} \stackrel{\leftrightarrow}{\partial_{\nu}} \chi-m \bar{\chi} \chi-i v_{\mu} \bar{\chi} \gamma_{5} \stackrel{\leftrightarrow}{\partial^{\mu}} \chi
$$

is actually physically identical to the standard LI model of a free Majorana spinor. One may recall that regular spinors in the class 1 of the Lounesto's classification have eight degrees of freedom, whereas Majorana spinors have four degrees of freedom. Equation (9) is a constraint that reduces two degrees of freedom of the Dirac spinor. Moreover the lacking degree of freedom is counted when the $U(1)$ gauge is taken into account for the Dirac spinor, but not for the Majorana one [1].

A more natural mapping between spinors, alternatively to Eq. (9) would be the one leading Weyl spinors, that are a particular class of dipole spinors with $U(1)$ gauge symmetry, to Dirac regular spinors, what moreover enables Penrose flags to be attached to Weyl spinors. However the relationship between Dirac and Weyl spinors is widely explored throughout the literature of QFT. Hence, we opt to obtain a mapping between regular spinor fields and flag-dipole ones, since flag-dipole spinors are important solutions of the Dirac equation in $f(R)$, torsional, gravity [20]. In 
fact, consider regular and type 4 spinors respectively [8],

$$
\begin{gathered}
\chi=\left(\begin{array}{l}
a_{0} \\
a_{1} \\
a_{2} \\
a_{3}
\end{array}\right) \in \mathbb{C}^{4} \text {, with } a_{0} \neq-\frac{a_{1} a_{2} a_{3}^{*}}{\left\|a_{2}\right\|^{2}} \\
\psi=\left(\begin{array}{l}
c_{0} \\
c_{1} \\
c_{2} \\
c_{3}
\end{array}\right)=\left(\begin{array}{c}
-\frac{c_{1} c_{2} c_{3}^{*}}{\left\|c_{2}\right\|^{2}} \\
c_{1} \\
c_{2} \\
c_{3}
\end{array}\right) \in \mathbb{C}^{4}, \text { with }\left\|c_{1}\right\|^{2} \neq\left\|c_{3}\right\|^{2} .
\end{gathered}
$$

The transformation which relates the spinor fields follows by taking $(v \cdot \Gamma) \chi=\lambda \gamma^{5} \chi=\psi-\chi$ in Eq. (9) [8]. Then

$$
\begin{aligned}
& \beta_{1}=1+\lambda=\frac{c_{2}}{a_{2}}=\frac{c_{3}}{a_{3}}, \\
& \beta_{2}=1-\lambda=\frac{c_{0}}{a_{0}}=\frac{c_{1}}{a_{1}},
\end{aligned}
$$

and it yields $\lambda=\frac{\beta_{1}-\beta_{2}}{2}$. In summary, we have shown how a general class of field redefinitions can relate between different classes of spinor fields in the LV and LI frameworks. The physical equivalence between models here described holds in the kinematical and dynamical classical aspects of these theories, leaving open the interesting question of the quantum equivalence. Second quantization of different classes of spinor fields can be accomplished, thus we believe the investigation of the quantum aspects of the relations exposed in this work is a viable and interesting topic to pursue.

\section{CONCLUDING REMARKS AND OUTLOOK}

We considered the impact of the inclusion of Lorentz Violation in theories involving general spinors in Riemann-Cartan spacetime. Couplings between the spinor bilinear covariants and torsion have been proved to provide a suitable signature to probe the sensitiveness of singular spinor fields to LV parameters. Specifically, we proved that flagpole spinor fields do not admit minimal coupling with torsion, and couplings with mass dimension four coefficients are influenced by the choice of flagpoles spinors, instead of the usual Dirac spinors. We also proved that when flagpoles spinors are regarded, non-minimal couplings between torsion and SM fields are the only feasible option. We conclude that flagpole spinor fields are less sensitive to Lorentz violation. 
The mapping between different classes of spinors in LV and Lorentz covariant frameworks was also discusses, being a relevant computational tool in QFT in LV setup. Explicit examples on how to map Dirac spinor fields, in LV theories, into flagpole and flag-dipole spinors in Lorentz invariant theories, were explicitly worked out.

The consideration of general spinor fields, as classified in the Lounesto scheme, can lead to new dimensions in the exploration of new physics involving Lorentz violation, and torsional gravity. 30]. As an example, since the eigenspinors of the charge conjugation operator with dual helicity [4] are also examples of flagpoles spinors, with the same structure as the flagpole in Eq. (37), phenomenological aspects in [10] can be further explored in the context of LV scenarios [5].

Acknowledgements. This work was partially supported by Conselho Nacional de Desenvolvimento Científico e Tecnológico (CNPq), Fundacão de Amparo à Pesquisa do Estado de São Paulo (FAPESP), via the following grants: CNPq 482874/2013-9, FAPESP 2013/22079-8 and 2014/24672-0 (AFF), FAPESP 2015/10270-0, CNPq 303293/2015-2 (RR), and Universidade Federal do ABC - UFABC PhD grant (JA).

\section{APPENDIX: THE COMPLETE EXPRESSION OF $\Omega_{\sigma}$}

For the sake of completeness, we quote here the complete expression for the function $\Omega_{\sigma}$ appearing in (34),

$$
\begin{aligned}
\Omega_{\sigma} & =\Delta\left\{\tilde{B}_{\mu} \partial^{\mu} \chi^{\dagger}+i \theta \tilde{B}_{\mu} \partial^{\mu} \chi^{\dagger}+i \tilde{B}_{\mu} \tilde{C}_{\alpha} x^{\alpha} \partial^{\mu} \chi^{\dagger}\right\} \gamma_{5} \gamma_{0} \chi \\
& +\left\{\chi^{\dagger}+\chi^{\dagger}(v \cdot \Gamma)^{\dagger}-i \tilde{C}_{\mu} x^{\mu} \chi^{\dagger}+\Delta\left(B_{\mu} \partial^{\mu} \chi^{\dagger}+\tilde{B}_{\mu} \partial^{\mu} \chi^{\dagger} \gamma_{5}+C_{\mu \nu} x^{\mu} \partial^{\nu} \chi^{\dagger}\right)-i \theta^{*} \chi^{\dagger}\right\} \gamma_{0} v \cdot \Gamma \chi \\
& +\left\{\chi^{\dagger}(v \cdot \Gamma)^{\dagger}+\Delta\left(B_{\mu} \partial^{\mu} \chi^{\dagger}+C_{\mu \nu} x^{\mu} \partial^{\nu} \chi^{\dagger}\right)+i \theta \chi^{\dagger}(v \cdot \Gamma)^{\dagger}+i \tilde{C}_{\alpha} x^{\alpha} \chi^{\dagger}(v \cdot \Gamma)^{\dagger}\right. \\
& \left.+i \theta \Delta\left(B_{\mu} \partial^{\mu} \chi^{\dagger}+i \tilde{C}_{\alpha} B_{\mu} x^{\alpha} \partial^{\mu} \chi^{\dagger}+i \theta C_{\mu \nu} x^{\mu} \partial^{\nu} \chi^{\dagger}+i C_{\mu \nu} x^{\mu} \tilde{C}_{\alpha} x^{\alpha} \partial^{\nu} \chi^{\dagger}\right)\right\} \gamma_{0} \chi \\
& +\left\{\tilde{B}_{\alpha} \chi^{\dagger}+\Delta\left(\tilde{B}_{\alpha} \chi^{\dagger}(v \cdot \Gamma)^{\dagger}-\tilde{C}_{\mu} x^{\mu} \tilde{B}_{\alpha} \chi^{\dagger}\right)+B_{\mu} \tilde{B}_{\alpha} \partial^{\mu} \chi^{\dagger}-\tilde{B}_{\mu} B_{\alpha} \partial^{\mu} \chi^{\dagger}\right. \\
& \left.+\tilde{B}_{\mu} \tilde{B}_{\alpha} \partial^{\mu} \chi^{\dagger} \gamma_{5}-\Delta \tilde{B}_{\mu} x^{\beta} C_{\beta \alpha} \partial^{\mu} \chi^{\dagger}+\tilde{B}_{\alpha} C_{\mu \nu} x^{\mu} \partial^{\nu} \chi^{\dagger}-i \theta^{*} \Delta \tilde{B}_{\alpha} \chi^{\dagger}\right\} \gamma_{0} \gamma_{5} \partial^{\alpha} \chi \\
& +\left\{B_{\alpha} \chi^{\dagger}+C_{\beta \alpha} x^{\beta} \chi^{\dagger}-i \tilde{C}_{\mu} \Delta C_{\beta \alpha} x^{\mu} x^{\beta} \chi^{\dagger}+B_{\mu} B_{\alpha} \partial^{\mu} \chi^{\dagger}+B_{\mu} C_{\beta \alpha} x^{\beta} \partial^{\mu} \chi^{\dagger}\right. \\
& +B_{\alpha} C_{\mu \nu} x^{\mu} \partial^{\nu} \chi^{\dagger}+C_{\mu \nu} C_{\beta \alpha} x^{\mu} x^{\beta} \partial^{\nu} \chi^{\dagger}-i \Delta\left(\theta^{*} B_{\alpha} \chi^{\dagger}-i \theta^{*} C_{\beta \alpha} x^{\beta} \chi^{\dagger}\right. \\
& \left.\left.+B_{\alpha} \chi^{\dagger}(v \cdot \Gamma)^{\dagger}+C_{\beta \alpha} x^{\beta} \chi^{\dagger}(v \cdot \Gamma)^{\dagger}-i \tilde{C}_{\mu} B_{\alpha} x^{\mu} \chi^{\dagger}\right)\right\} \gamma_{0} \partial^{\alpha} \chi
\end{aligned}
$$


where $\Delta$ encompasses the sign regarding the hermitian conjugation. The remaining $\Omega$ functions appearing in (36) are obtained from the previous equations after the identifications indicated in (36).

[1] P. Lounesto, "Clifford Algebras and Spinors", Cambridge Univ. Press, Cambridge, 2002.

[2] J. Vaz and R. da Rocha, "An Introduction to Clifford Algebras and Spinors", Oxford University Press, Oxford, 2016.

[3] J. M. Hoff da Silva and R. da Rocha, Phys. Lett. B 718 (2013) 1519.

[4] D. V. Ahluwalia, C. -Y. Lee, and D. Schritt, Phys. Rev. D 83 (2011) 065017.

[5] C. Y. Lee, Phys. Lett. B 760 (2016) 164.

[6] C. H. C. Villalobos, J. M. H. da Silva, and R. da Rocha, Eur. Phys. J. C 75 (2015) 266.

[7] A. E. Bernardini and R. da Rocha, Phys. Lett. B 717 (2012) 238.

[8] R. T. Cavalcanti, Int. J. Mod. Phys. D 23 (2014) 1444002.

[9] L. Fabbri, Int. J. Geom. Meth. Mod. Phys. 13 (2016) 1650078.

[10] M. Dias, F. de Campos and J. M. Hoff da Silva, Phys. Lett. B 706 (2012) 352.

[11] R. da Rocha and J. M. Hoff da Silva, Europhys. Lett. 107 (2014) 50001.

[12] L. Fabbri, Phys. Rev. D 85 (2012) 047502.

[13] S. Vignolo, L. Fabbri and C. Stornaiolo, Phys. 524 (2012) 826.

[14] R. Casana, V. Y. Fainberg, J. T. Lunardi, B. M. Pimentel and R. G. Teixeira, Class. Quant. Grav. 20 (2003) 2457.

[15] L. Fabbri and S. Vignolo, Class. Quant. Grav. 28 (2011) 125002.

[16] L. Fabbri, Gen. Rel. Grav. 46 (2014) 1663.

[17] A. Pinho S. S., S. H. Pereira, J. F. Jesus, Eur. Phys. J. C 75 (2015) 36.

[18] L. Bonora, K. P. S. de Brito, and R. da Rocha, JHEP 1502 (2015) 069.

[19] L. Bonora and R. da Rocha, JHEP 1601 (2016) 133.

[20] R. da Rocha, L. Fabbri, J. M. Hoff da Silva, R. T. Cavalcanti, and J. A. Silva-Neto, J. Math. Phys. 54 (2013) 102505.

[21] V. A. Kostelecký, N. Russell and J. Tasson, Phys. Rev. Lett. 100 (2008) 111102.

[22] D. Colladay and V. A. Kostelecký, Phys. Rev. D 55 (1997) 6760.

[23] D. Colladay and V. A. Kostelecký, Phys. Rev. D 58 (1998) 116002.

[24] V. A. Kostelecky, Phys. Rev. D 69 (2004) 105009.

[25] A. E. Bernardini, Phys. Rev. D 75 (2007) 097901.

[26] B. Charneski, M. Gomes, R. V. Maluf and A. J. da Silva, Phys. Rev. D 86 (2012) 045003.

[27] R. V. Maluf, J. E. G. Silva and C. A. S. Almeida, Phys. Lett. B 749 (2015) 304.

[28] M. A. Anacleto, F. A. Brito, and E. Passos, Phys. Lett. B 694 (2010) 149.

[29] C. Miller, R. Casana, M. M. Ferreira Jr., and E. da Hora, Phys. Rev. D 86 (2012) 065011. 
[30] M. Gomes, T. Mariz, J. R. Nascimento, E. Passos, A. Y. Petrov and A. J. da Silva, Phys. Rev. D 78 (2008) 025029.

[31] A. E. Bernardini and R. da Rocha, Phys. Rev. D 75 (2007) 065014.

[32] D. Bazeia, M. M. Ferreira Jr., A. R. Gomes, and R. Menezes, Physica D 239 (2010) 942.

[33] E. A. Notte-Cuello, W. A. Rodrigues Jr., Q. A. G. de Souza, Rept. Math. Phys. 60 (2007) 135.

[34] D. Colladay and P. McDonald, J. Math. Phys. 43 (2002) 3554.

[35] G. Velo, D. Zwanziger, Phys. Rev. 186 (1969) 1337.

[36] G. Velo, D. Zwanziger, Phys. Rev. 188 (1969) 2218.

[37] L. Fabbri, Mod. Phys. Lett. A 26 (2011) 2091.

[38] L. Fabbri, Int. J. Theor. Phys. 51 (2012) 954.

[39] S. Deser and B. Zumino, Phys. Lett. B 62 (1976) 335.

[40] S. Deser and A. Waldron, Nucl. Phys. B 631 (2002) 369.

[41] L. F. Urrutia and J. D. Vergara, Phys. Rev. D44 (1991) 3882.

[42] L. Fabbri, Annales Fond. Broglie 33 (2008) 365.

[43] I. L. Shapiro, Phys. Rep. 357 (2002) 113.

[44] A. F. Ferrari, M. Gomes, J. R. Nascimento, E. Passos, A. Y. Petrov and A. J. da Silva, Phys. Lett. B $652(2007) 174$.

[45] R. da Rocha and J. M. Hoff da Silva, Adv. Appl. Clifford Algebras 20 (2010) 847. 